\title{
A Framework for Constrained Control Allocation Using CFD-based Tabular Data
}

\author{
A. Da Ronch $*$ M. Ghoreyshi ${ }^{\dagger}$ D. Vallespin ${ }^{\ddagger}$ K.J. Badcock $§$ \\ Department of Engineering, University of Liverpool, Liverpool \\ England L69 3BX, United Kingdom \\ Z. Mengmeng 『 J. Oppelstrup ॥ \& A. Rizzi ** \\ Royal Institute of Technology (KTH) \\ Stockholm, 100 44, Sweden
}

This paper describes a framework for control allocation problem using Computational Fluid Dynamics (CFD) aerodata, which is represented by a multidimensional array of dimensionless coefficients of aerodynamic forces and moments, stored as a function of the state vector and control-surface deflections. The challenges addressed are, first, the control surface treatment for the automated generation of aerodata using CFD and, second, sampling and data fusion to allow the timely calculation of large data tables.

In this framework, the generation of aerodynamic tables is described based on an efficient sampling/data fusion approach. Also, the treatment of aerodynamics of control surfaces is being addressed for three flow solvers: TORNADO, a vortex-lattice method, and two CFD codes, EDGE from the Swedis Defence Agency and PMB from the University of Liverpool. In TORNADO, the vortex points located at the trailing edge of the flaps are rotated around the hinge line to simulate the deflected surfaces. The transpiration boundary conditions approach is used for modeling moving flaps in EDGE, whereas, the surface deflection is achieved using mode shapes in PMB.

The test cases used to illustrate the approaches is the Ranger 2000 fighter trainer and a reduced geometry description of Boeing 747-100. Data tables are then generated for the state vector and multiple control surface deflections. The look-up table aerodata are then used to resolve the control allocation problem under the constraint that each surface has an upper and lower limit of deflection angle.

\section{Introduction}

In traditional aircraft design, a single controller is associated with each rotational degree of freedom to control the attitudes of the airplane. Elevator is used as the primary control of the pitch attitude and the aileron and rudder deflections control the roll and yaw angles, respectively. Typically, a positive elevator deflection results in a negative pitching moment, a positive aileron deflection will result in a negative rolling moment and a positive rudder will induce a negative yawing moment. In this situation, the system is square,

\footnotetext{
*Ph.D. Student, AIAA Member

${ }^{\dagger}$ Research Assistant, AIAA Member

$\ddagger$ Ph.D. Student, AIAA Member

$\S$ Professor, SMAIAA

I $\mathrm{PhD}$ student

\| Senior Lecturer

** Professor, SMAIAA
} 
i.e. the number of control surfaces and moments are equal, and the solution of the control allocation is unique.

Modern combat and civilian aircraft employ more than three primary control surfaces, mainly to maximize mission performance and enhance survivability in the presence of control surface failure(s). The number of surfaces can reach up to $20 .^{1}$ These surfaces are effective up to a certain limits of physical deflection and might be employed independently and generate forces and moments in all three aircraft body axis. Such an airplane is referred to as a redundant system, a system which has more inputs than outputs. ${ }^{2}$ Aircraft control allocation problem is finding an optimum allocation of controls that maps the desired moments from the aircraft control system to airplane surfaces deflections by avoiding control surface deflection saturation. ${ }^{1}$ The other names of this problem are control management, control distribution and control selection. ${ }^{2}$ The solution of this problem helps to maintain the aircraft performance in the presence of a control surface failure $^{3}$ The control allocation now plays an important role within aircraft flight control system whose number of output commands are smaller than controls. Fig. 1 shows architecture of an aircraft autopilot employing surface allocation algorithm. The desired set of moments are specified by the aircraft control algorithm are fed to the surface allocation algorithm that finds the allocated surface deflections.

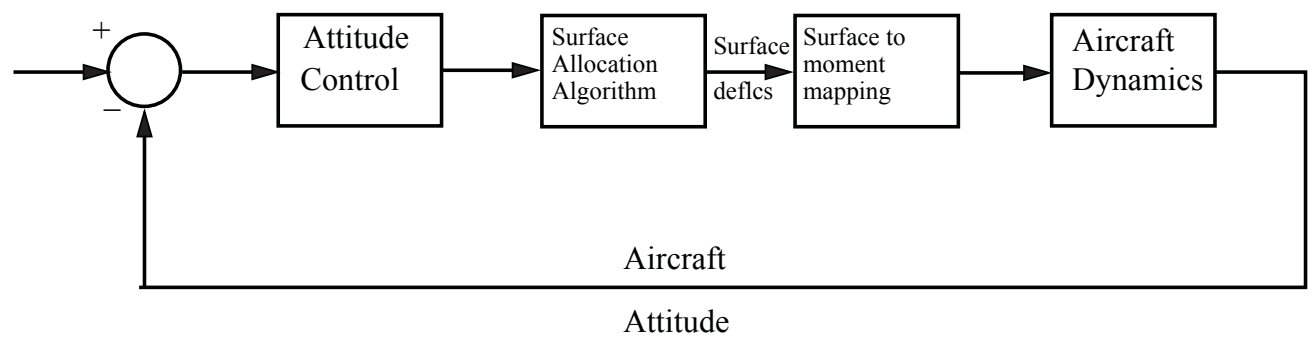

Figure 1. Aircraft autopilot architecture ${ }^{1}$

The formulation of control allocation is a constrained multiple optimization type that needs to be solved numerically. Extensive control allocation algorithms have been developed over the past two decades. ${ }^{4-9}$ For example, Durham ${ }^{4}$ presented a direct approach that includes three steps: a) the attainable moment subset is determined from the limits of control surfaces, b) the boundaries of the subset are then found and c) a vector of control surface that maps to the points within or on that boundary defines the solution of control allocation problem. Bodson ${ }^{6}$ compared different algorithms including simplex and interior-point methods. Also, the performance of many open and closed loop linear control allocation algorithms were presented by Page and Steinberg. ${ }^{8}$

Many allocation methods are based on the stability derivative approach assuming that the aircraft moments are linear function of control surfaces, which may be valid locally. In the occurrence of one or more failed control surfaces, the remaining surfaces might be pushed to the limits of deflection angles, where, the variation of moments with deflection can be highly nonlinear. ${ }^{10}$ In conceptual design, the application of CFD to modeling aerodynamics of large surface deflections and unconventional control surfaces provide quite accurate results in the absence of experimental data. This paper examines the use of CFD generated tabular data and genetic algorithm for solution of the control allocation.

Today there is an increasing interest to run CFD for the aerodynamics calculations of aircraft designs earlier in their definition, thus helping with early design of the control-system. The aerodynamic model considered in this paper is tabular in form. Tables, in contrast to stability derivatives, are not linearized, and are consistent with quasi-steady aerodynamics for a wide range of flight conditions. But a large number, in order of 30 thousand solutions, ${ }^{11}$ of table entries must be filled. This database increases by increasing the number of control surfaces. The brute-force calculation of the entire table using CFD is not feasible due to computational cost. Sampling and data fusion methods have been proposed to generate aerodynamic tables including the aircraft states and the controls of the pitch, roll and yaw rotations. ${ }^{12}$

This paper looks further into the CFD modeling of aircraft aerodynamics with multiple control surfaces and describes an efficient sampling/data fusion for such a case. Also, the treatment of aerodynamics of control surfaces is addressed for the vortex lattice method TORNADO and for two CFD flow solvers, EDGE and PMB. The look-up table aerodata are then used to find the solution of control allocation problem for a reduced geometry order of B747 aircraft and Ranger 2000 trainer. 


\section{Description of CEASIOM, Aircraft Design Code}

The Computerized Environment for Aircraft Synthesis and Integrated Optimization Methods (CEASIOM, www.ceasiom.com $)^{13}$ has been used as the design code used in the current paper. The geometry handling and aerodynamic module of the code are described:

\section{A. Geometry}

The CEASIOM aircraft parameters include definitions of lifting surfaces, fuselage, wing-fuselage fairing, ventral fin, engines, fuel tanks, baggage hold and cabin dimensions. The definition also covers cranked lifting surfaces, multiple lifting surfaces with any number of leading and trailing edge moving devices. The aerodynamic module in CEASIOM converts the aircraft description into the native geometry format for the aerodynamic prediction tools.

Of particular interest is the approach used to automatic mesh generation for the Euler solver. The surface modeling package, SUMO ${ }^{a}$, produces a surface model, and its triangulation. SUMO is a rapid geometry modeling tool for parametrically-defined aircraft configurations. The code, written in $\mathrm{C}++$, has a library of geometric primitives based on B-spline curves and surfaces to create a parameterized watertight surface model of the complete XML-aircraft. The automatic mesh generation tool in SUMO provides an unstructured surface mesh. The mesh control parameters are estimated from the model geometrical features, such as radii of curvature and the presence of sharp edges. From the surface mesh, unstructured volume meshes can be automatically generated using the tetrahedral mesh generator TetGen ${ }^{\mathrm{b}}$.

\section{B. Aerodynamics}

The aerodynamic model considered in this paper is tabular in form. The model consists of tables of forces and moments for a set of aircraft states and controls (e.g. aircraft angle of attack, side-slip angle, control deflections, etc.), which spans the flight envelope. This potentially entails a large number of calculations, which will be a particular problem due to the computational cost if CFD is the source of the data. This issue has been addressed by sampling and reconstruction based on Kriging interpolation and data fusion using Co-Kriging as described in reference. ${ }^{12}$

Two scenarios were considered, based on (1) a requirement for the generation of static table that include the table of Mach number and angles of attack and side-slip and (2) for updating the static table for control tables of elevator, rudder and aileron. Both approaches are available in the Computerized Environment for Aircraft Synthesis and Integrated Optimization Methods, CEASIOM, computer code.

In the first scenario it is assumed that the static table is generated without user intervention. The emphasis is on a sampling method which identifies the nonlinearities in the force and moment tables with respect to the static aircraft state parameters. Approaches to the sampling based on the Mean Squared Error (MSE) criterion of Kriging and the Expected Improvement Function (EIF) were considered. ${ }^{12}$

In the second scenario, it is assumed that the control tables are incremented from the static table, i.e. the static table represents the main trends of aerodynamic forces and moments, while the controls result in the increments of these trends. Data fusion based on Co-Kriging is then used to update the static table, based on a small number of calculations of control surface deflections.

Using these techniques it was shown that tables which are practically useful could be generated in the order of sixty calculations under the first scenario and ten calculations under the second scenario.

The CEASIOM aerodynamic module includes a variety of sources of aerodynamic data and can be connected easily to an external solver. ${ }^{14}$ The solvers used are:

- Potential Solver

The potential flow solver, TORNADO, can predict a wide range of aircraft stability and control aerodynamic derivatives ${ }^{\mathrm{c}}$.

The original implementation of the vortex lattice method models the lifting surfaces only. Research has been addressed in order to represent the fuselage effect, thus modifying and extending the original

\footnotetext{
ahttp://www.larosterna.com/sumo.html

${ }^{b}$ http://tetgen.berlios.de/

${ }^{\mathrm{c}}$ www.ave.kth.se/divisions/aero/software/tornado/tornado.html
} 
baseline implementation. A review of alternative methods to approximate the aerodynamic contributions generated around non-slender bodies was given in Khrabrov et al. ${ }^{15}$ The capabilities of a modified implementation of TORNADO, which models non-slender bodies and take into account for viscous contributions, were presented in a recent study. ${ }^{16}$ In the context of this paper, the fuselage effect is not calculated and the flow approximated as inviscid. The effects of compressibility at high Mach number are estimated by the Prandtl-Glauert similarity role (see Anderson ${ }^{17}$ ).

For the control surface deflections, the vortex points located at the trailing edge of the flap are rotated around the hinge line. This causes a motion where the vortex segments on the flap and in the wake changes direction slightly. ${ }^{18}$

- EDGE

EDGE $^{19}$ is a parallelized CFD package developed by FOI. The code can be applied to $2 \mathrm{D} / 3 \mathrm{D}$ viscous (RANS) or inviscid (Euler), compressible flow problems on unstructured grids with arbitrary elements and is used in Euler mode in CEASIOM. Also, EDGE allows both steady state and time accurate calculations.

The space discretisation exploits a node-centred finite-volume technique using an edge based data structure. The computational elements are a set of non-overlapping cells formed as the dual of the primary tetrahedral mesh. Explicit Runge-Kutta time stepping integrates the discrete equations in time. Accelerated convergence to steady state is promoted using agglomeration multigrid and implicit residual smoothing.

The way EDGE calculates the aerodynamics of control surface deflections is based on the use of transpiration boundary conditions. In this approach, instead of moving the grid, the wall velocity component normal to the actual deflected surface is prescribed. Such approach eliminates the need of mesh deformation, thus all calculations can be run virtually on the clean configuration grid, but on the other hand, this imposes a limitation on the amount of maximum and minimum deflections. In this paper, EDGE is used in the Euler mode with pre-set reasonable values for the maximum and minimum deflection of each defined control surface.

- $\mathrm{PMB}$

The flow solver used for this study is the University of Liverpool PMB (Parallel Multi-Block) solver. The Euler and RANS equations are discretised on curvilinear multi-block body conforming grids using a cell-centred finite volume method which converts the partial differential equations into a set of ordinary differential equations. The equations are solved on block structured grids using an implicit solver. A wide variety of unsteady flow problems, including aeroelasticity, cavity flows, aerospike flows, delta wing aerodynamics, rotorcraft problems and transonic buffet have been studied using this code. More details on the flow solver can be found in Badcock et al. ${ }^{20}$ and a validation against flight data for the F-16XL aircraft is made in reference. ${ }^{21}$

The control surfaces are blended into the geometry in the current work following the approach given in reference. ${ }^{22}$ After the surface grid point deflections are specified, transfinite interpolation is used to distribute these deflections to the volume grid.

\section{Control Allocation Problem}

\section{A. Problem Statement}

Aircraft can be considered as a dynamic system with a mathematical model in the state space given by:

$$
\dot{x}=f(x(t), u(t))
$$

and an algebraic output equation as:

$$
y(t)=h(x(t))
$$

where $x \subset \Re^{p}$ is column vector of internal state variables or uncommanded states, $u \subset \Re^{q}$ is column vector of the input to system variables or the system controls and $y \subset \Re^{r}$ is the column vector of system outputs or commanded states. Here, the aircraft equations of motion represent the state space function, with positions, 
attitudes, linear and angular velocity components as internal state variables $x$. The control surface deflections are the inputs and the aerodynamic forces and moments are assumed as the system output, that results in $r=6$. For a linear system, the equations are changed to:

$$
\dot{x}=A x+B u, \quad y(t)=C x
$$

Since we are concerned to control the aircraft attitude, we only consider aero moment coefficients, i.e. $r=3$ and name it $m=\left[C_{l}, C_{m}, C_{n}\right]$, where the vector contents show the moments for roll, pitch and yaw axis. A mapping exists between the moment vector and the inputs, i.e. :

$$
m=D u
$$

where $D$ is the control effectiveness matrix. If $q>3$, the matrix $D$ is not square and finding $u$ from Eq. 6 for a given $m$ requires solving equations with more unknown than equations. ${ }^{6}$ Although, such a solution might seem straightforward, but the difficulty arises when the vector $u$ is bounded to an upper and lower limits. The constraints for each control $i$ is given in the form of:

$$
u_{\min , i} \leq u_{i} \leq u_{\max , i} \quad \text { for } \quad i=1,2, . ., q
$$

the constraints avoid the saturation of control surface deflection. For CFD-based aerodata, we consider a non-linear system, where the mapping of the moment coefficients and control surfaces are in general form of:

$$
m=g(u)
$$

where $g$ is a smooth function. In this paper, the look-up tables are used to represent the function $g$.

The allocation problem is fining $u^{*}$ of a system with mapping equations of 6 for a desired moment vector, $m_{d}$ and subject to constraints of 5 .

\section{B. Solution Algorithm}

The linear and quadratic programming methods are two typical examples of solving the problem. A linear method is used in this paper. Given the desired moment $m_{d}$, find a vector $u$ such that

$$
j=\left\|g(u)-m_{d}\right\|
$$

is minimized subject to 5 . For solving this problem, we use genetic algorithm tools available in MATLAB. The genetic algorithm helps to find a global minimum point. However, the computational cost might not be attractive for the use in aircraft onboard computers. A suggestion is to find a mapping between the optimum solutions and several training data and learn the mapping function via neural networks or surrogate model. The model is then run fast to approximate the optimum solution.

\section{Test Cases}

\section{A. Boeing 747-100}

The configuration used in the current paper for testing is based on the Boeing 747-100 aircraft (Fig. 2). For a computer-aided analysis and optimization, the geometry of the initial concept must be described. The design code used in the current paper, CEASIOM, incorporates a parameterized description of the geometry, named in this paper the XML-aircraft. The B747 XML-aircraft, that is defined by around 100 parameters, lacks the details of fuselage upper deck and the wing/fusleage fairing. From this XML description, an unstructured mesh was generated for the Euler solver using SUMO. With respect to the control surfaces, SUMO again was used to define the position and dimensions of surfaces.

The geometric properties of the B747 model are summarized in Table 1. The model centre of gravity is located at $45.6 \%$ of the fuselage total length from the foremost point.

The B747 wing has triple-slotted trailing flaps and Krueger leading edge flaps. The Krueger flaps outboard of the inboard nacelle are variable cambered and slotted while the inboard Krueger flaps are standard unslotted. Longitudinal control is obtained through four elevator segments and a movable stabilizer. The lateral control employs five spoiler panels, an inboard aileron between the inboard and outboard flaps, and an outboard aileron which operates with flaps down only on each wing. The five spoiler panels on each 


\begin{tabular}{ll}
\hline \hline Length Overall (m) & 68.5 \\
Wing Span (m) $^{(m)}$ & 59.64 \\
Wing Area m $^{2}$ & 511 \\
Mean Aerodynamic Chord (MAC) (m) & 9.0798 \\
\hline \hline
\end{tabular}

Table 1. Reference values of the B747 model geometry

wing also operate symmetrically as speed breaker in conjunction with the most inboard sixth spoiler panel. Directional control is obtained from two rudder segments. The considered control surfaces are shown in Fig. 2. Also, the Tornado geometry of the Boeing 747-100 is shown in Fig. 3. The model consists of only lifting surfaces with moving flaps. For illustration purposes, all flaps are deflected to their maximum angle.

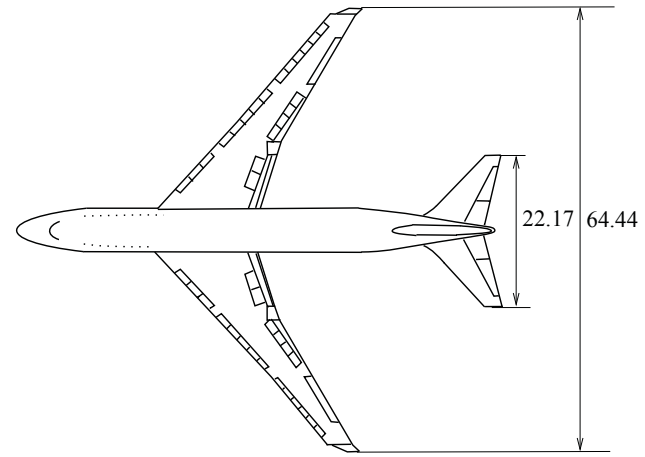

Length unit in meter
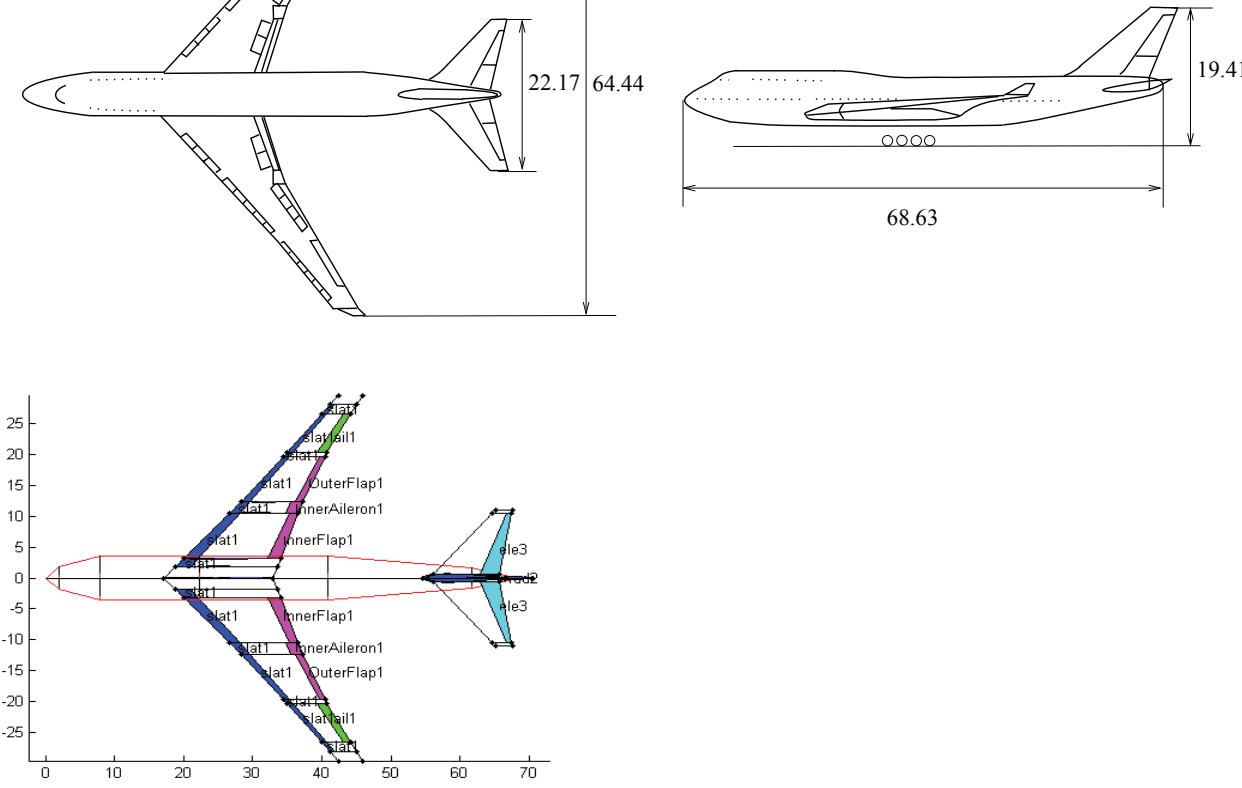

Figure 2. B747 geometry with control surfaces

From the XML description of Boeing 747-100, a CAD model was automatically constructed using SUMO. This lacks refinements like the fuselage/wing junction blending of actual aircraft. The EDGE meshes were generated automatically by SUMO. The unstructured mesh consists of 257956 nodes, 1694280 edges and 1348165 tetrahedra. Twenty boundaries are defined in the grid, including the farfield and eleven boundaries pertaining to the defined control surfaces. A zero normal velocity is imposed on all non-deflected surfaces. Fig. 4 shows the CAD model with all control surfaces and the generated mesh.

\section{B. Ranger 2000}

Ranger 2000 is a mid-wing, tandem seat training aircraft powered by one turbofan engine with uninstalled thrust of $14190 \mathrm{~N}$. A three-view of the vehicle is shown in Fig. 5. Also, the general dimensions of aircraft are listed in Table 2.

The vehicle flight control system system consists of three conventional control surfaces: The elevator at the tail, the rudder at the fin and left and right ailerons (Fig. 6). The aircraft has also a split flap. The position limits of each surface is given in Table 3 , with positive angles indicating the elevator and flap trailing edge down, the left aileron trailing edge down and the rudder trailing edge toward the left wing (see Fig. 6). 


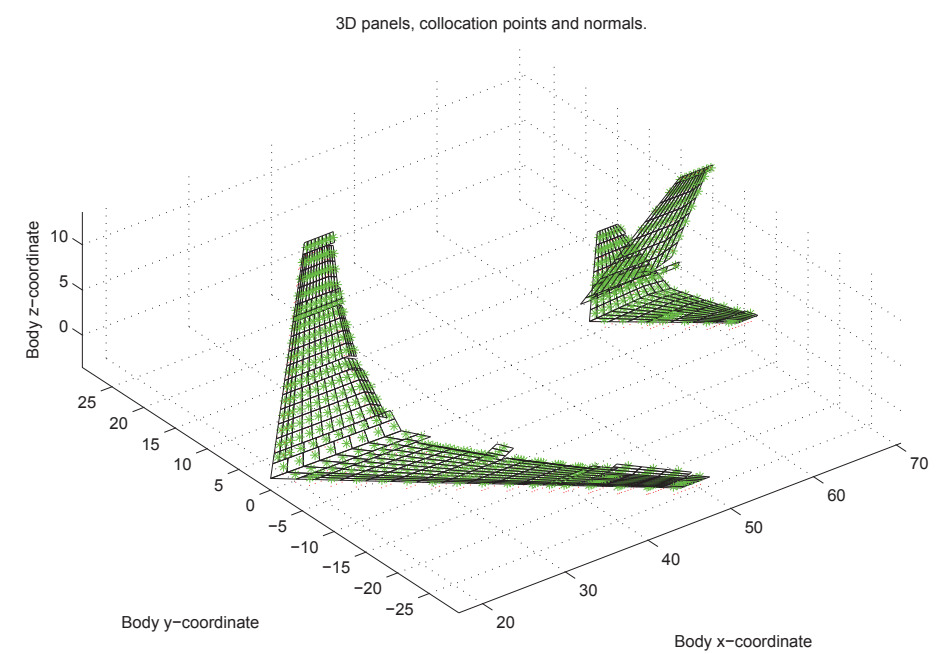

Figure 3. B747 TORNADO aerodynamic panels
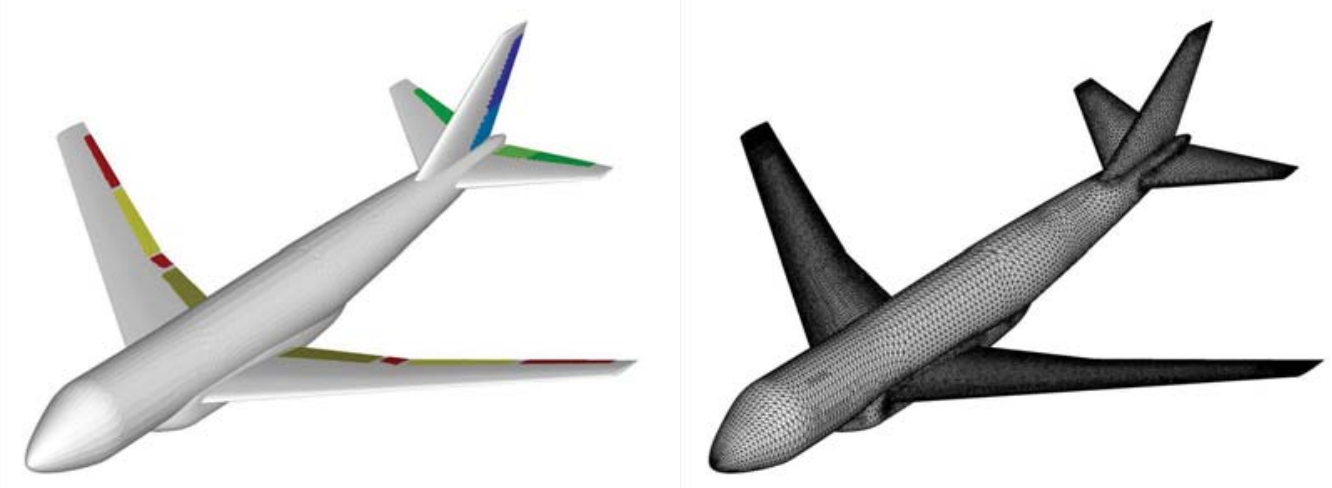

Figure 4. SUMO geometry and generated mesh for the B747

\begin{tabular}{|c|c|}
\hline Length Overall (m) & 10.39 \\
\hline Wing Span (m) & 10.46 \\
\hline Height Overall (m) & 3.58 \\
\hline Wing Area $\mathrm{m}^{2}$ & 15.5 \\
\hline Mean Aerodynamic Chord (MAC) (m) & 1.545 \\
\hline Wing Taper Ratio & 0.519 \\
\hline
\end{tabular}

Table 2. General Dimensions of Ranger 2000

A multiblock structured grid was generated using the commercial grid generation tool ICEMCFD. Both full and half configurations were generated, the latter to save on computing costs in the simulation of longitudinal flight dynamics. The lateral flight dynamics tables, on the other hand, required the full model flow prediction. The half configuration has 14.5 million points arranged in 2028 blocks.

Fig. 7 (a) shows the overall view of the meshed geometry. Fig. 7 (b) and (c) show the diamond shaped 

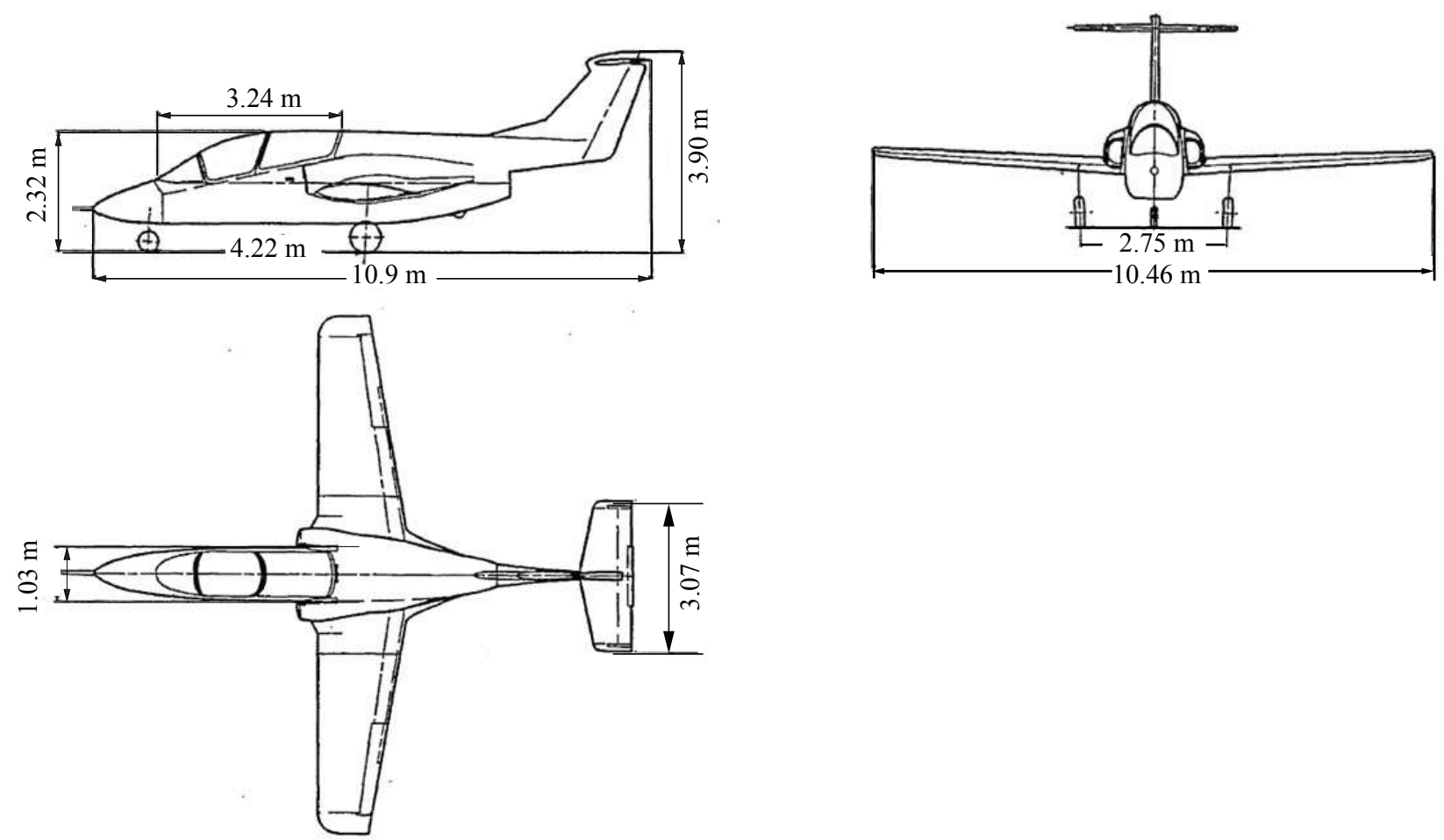

Figure 5. Three-Views of Ranger 2000

\begin{tabular}{lll}
\hline \hline Elevator & $\delta_{\text {ele }}$ & $-25 /+15^{0}$ \\
Right Aileron & $\delta_{\text {ail }}$ & $-25 /+15^{0}$ \\
Left Aileron & $\delta_{\text {ail }}$ & $-25 /+15^{0}$ \\
Rudder & $\delta_{\text {rud }}$ & $\pm 17.5^{0}$ \\
Split Flap & $\delta_{\text {flp }}$ & $0 / 60^{0}$ \\
\hline \hline
\end{tabular}

Table 3. Control surface deflection limits

tip topology used to accommodate the cells around the main wing and tail-plane. The wing has a H-type topology around the leading edge to improve the cell quality in the wing-engine-fuselage junction while the horizontal tail-plane blocking consists of a C-type round its leading edge.

The aforementioned control surfaces are also included in the geometry (Fig. 6). These were the elevator, aileron and rudder which can be deflected for steady state calculations and during time accurate simulations. The half grid requires 1.5 hours on 128 processors on the United Kingdom's academic supercomputer (Cray inc) to obtain a fully converged steady state solution.

\section{Aerodynamic Results}

\section{Boeing 747-100}

A look-up table of aerodynamic data for B747-100 aircraft has been generated using TORNADO. The table represents values of aerodynamic forces and moments for varying incidence, side-slip, Mach number, and deflection angles. The range of incidence angle, Mach number and side-slip angle are $\left[-5^{0}, 15^{0}\right],[0.1,0.8]$, and $\left[-10^{0}, 10^{\circ}\right]$ respectively. The inner and outer aileron and rudder are limited to $\left[-17.5^{0}, 17.5^{0}\right]$. The inner and outer flaps are moved in the range of $\left[-15^{0}, 15^{0}\right]$, also the range of moving inboard and outboard elevator 


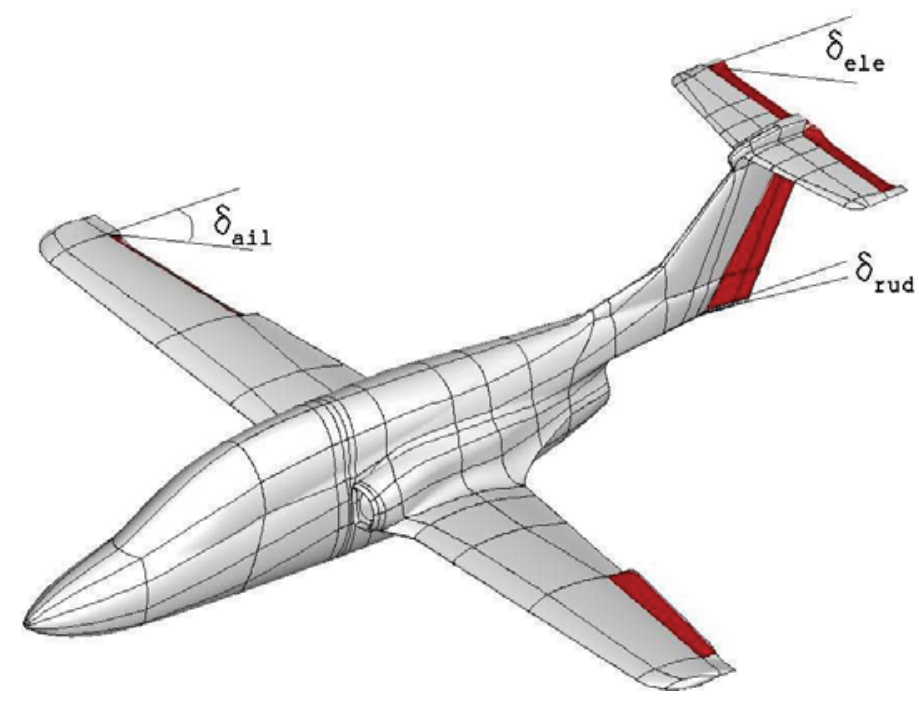

Figure 6. Ranger control surfaces- The configuration shows the positive deflection angles.
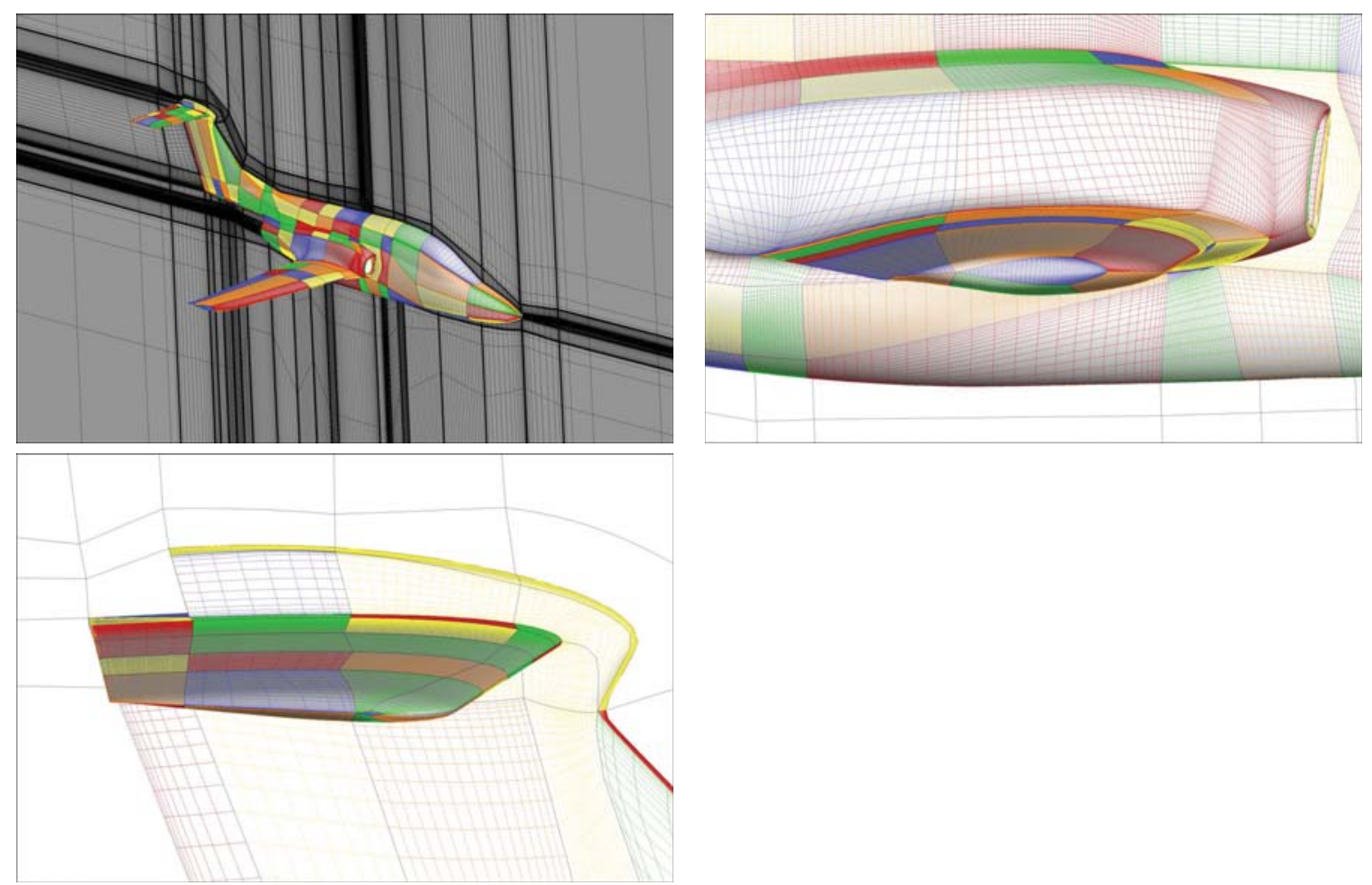

Figure 7. The Ranger grid 
is $\left[-20^{0}, 15^{0}\right]$.

Experimental data for the steady aerodynamics of Boeing 747-100 are available. The experiment conditions correspond to $M_{\infty}=0.1$ and $R e=4.59 \times 10^{6}$. The predictions of lift and pitching moment coefficients are compared with experimental data in Fig. 8. TORNADO predicts closely the linear slope of the lift coefficient, but the stall predictions are off due to inviscid assumption. The slope of pitching-moment is different from experimental data mainly due to lack of modeling fuselage. The fuselage often shifts aerodynamic center aftward.

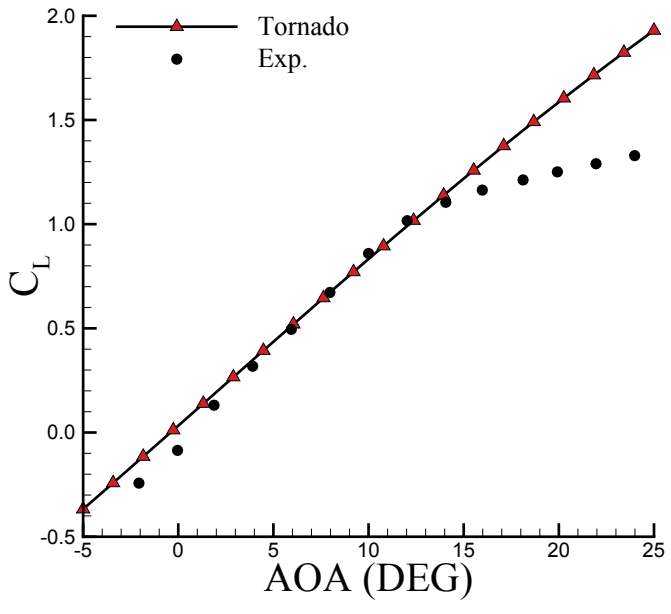

(a) Lift coefficient

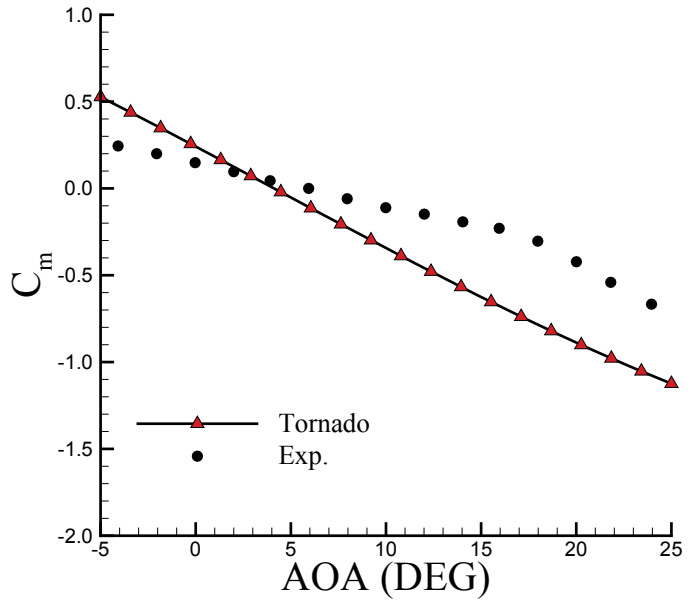

(b) Pitching moment coefficient

Figure 8. Prediction of lift and pitching moment coefficients for different number of panels at $M=0.1$

The main aerodynamic variables are taken to be the angle of attack and the Mach number. All forces and moments are assumed to depend on these variables in combination with each of the remaining variables separately. Hence, there are 15 three dimensional table need to be generated. Note that the right and left control surfaces are assumed to move separately. The sampling and fusion methods are applied to generate all tables using TORNADO. A 1,440 entry, three-parameter table (angle of attack, Mach number and sideslip angle) was first generated using TORNADO. An initial design of $4 \times 4 \times 4$ was defined. Next, sampling was used to define parameters for additional 64 calculations, and Kriging was then used to construct all of the table entries.

Fig. 9 shows the error convergence using sampling for lift and pitching and yawing moment coefficients. The figure shows that sampling performs well to represent the full table data. The initial and final samples are also shown in Fig. 10. For inviscid TORNADO, the sampling tends to fill the large gaps between the initial points. For the tables corresponding to control surface deflection, data fusion was used. The generated table of incidence, Mach number and side-slip angle describes the underlying behaviour of aerodynamic coefficients and control surface deflections can be considered as the increment of these trends. For each control surface, fifteen samples were only generated using Latin Hypercubic Sampling (LHS). The values at these samples were then used to increment the original table values using Co-Kriging.

The effects of inner and outer elevator and flap deflections on the lift and pitching moment coefficients are shown in Fig. 11. Figure shows that a downward deflection of all surfaces increase the lift coefficient, while the inner flap has the highest rate of changes. Also, for a downward inner flap deflection, the pitching moment increases due to location of flap relative to the aircraft aerodynamic center. All other controls result in a negative pitching moment increment for positive deflection angles. The inner elevator is the most powerful surface for pitching-moment control. Likewise, the effects of lateral devices into rolling and yawing moments are shown in Fig. 12. The figure shows that the most control effectiveness for the rolling and yawing moments are from the outer aileron and rudder, respectively.

For high speed aerodynamics, EDGE solver was used. A flight envelope was tailored around the trim condition of the B747 whilst in cruise at an altitude of $10670 \mathrm{~m}$. The Mach number was varied between 0.75 and 0.9 and the angle of incidence from $1^{\circ}$ up to $6^{\circ}$. The surface pressure distribution of two simulations 


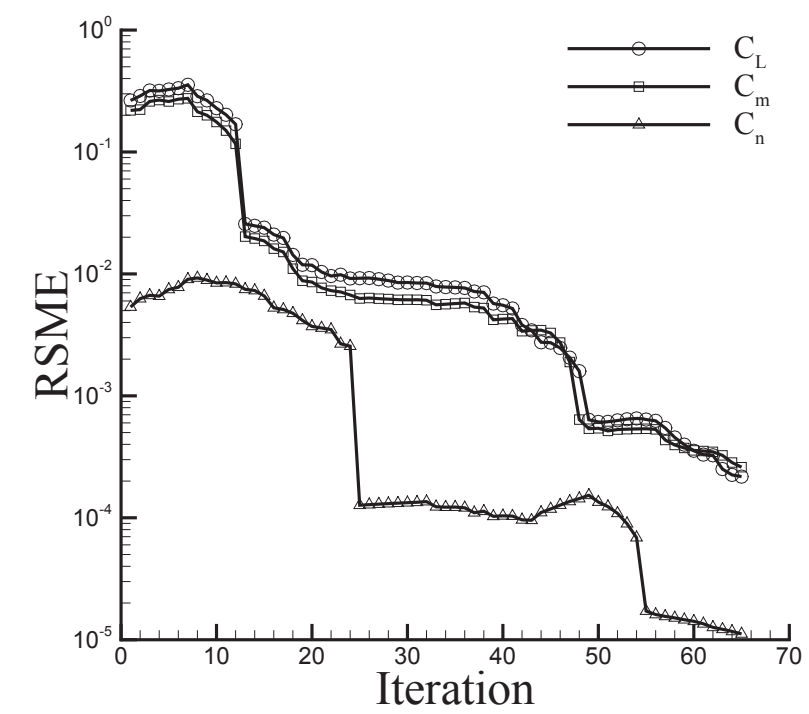

Figure 9. Error convergence

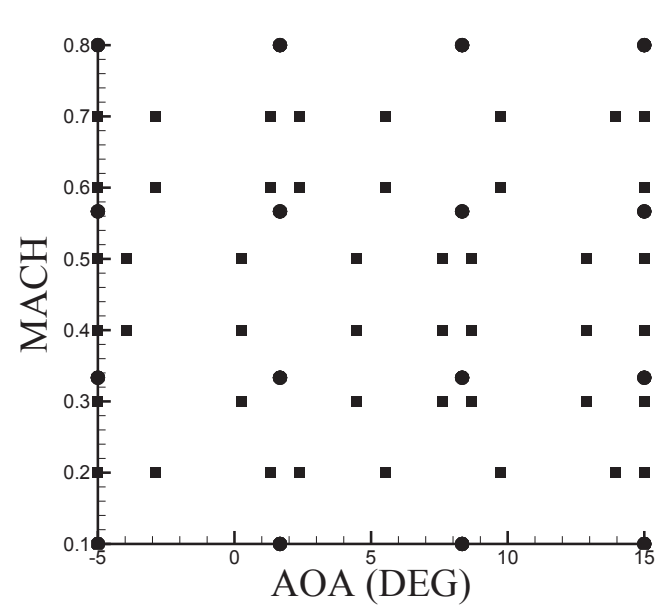

(a)

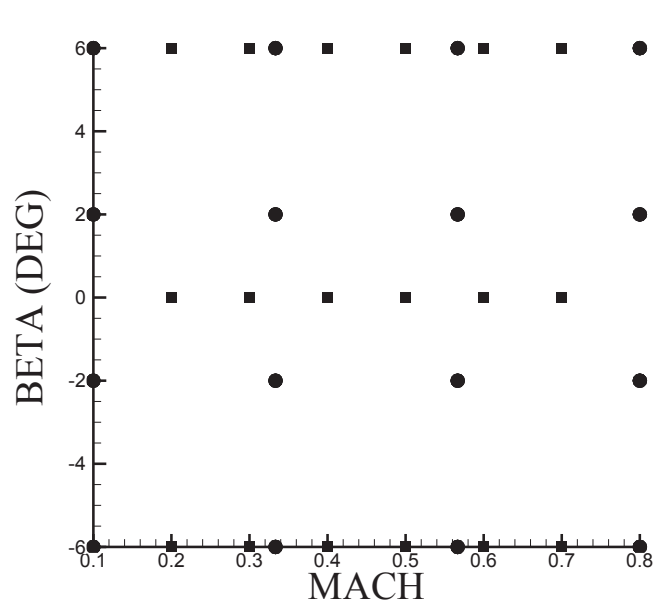

(b)

Figure 10. Samples- the circles denote the initial samples and the squares represent the samples generated by sampling approach

are shown in Fig. 13 (a) and (b). Figures show the shock waves are formed on the upper surfaces.

The transpiration boundary conditions approach was used to calculate the aerodynamics of moving surfaces. For examples, the solutions of a rudder and an inner aileron deflected configuration are shown in Fig. 13 (c) and (d), respectively. This approach eliminates the need to generate different grids for each configuration of deflected control surfaces, but such an approach is limited in terms of the amount of the maximum and minimum deflections, thus, the range of moving surfaces in EDGE is smaller than those used for Tornado. Beside, the way we defined the controls is to move the right and left surfaces together. Using an available baseline table for angle of attack, Mach number and side-slip and 15 additional calculations for each control surface, the control tables were generated using Co-Kriging. The changes in lift, yawing moment and rolling moment coefficients from control surface deflections are compared with TORNADO results in Fig.

11 of 18 


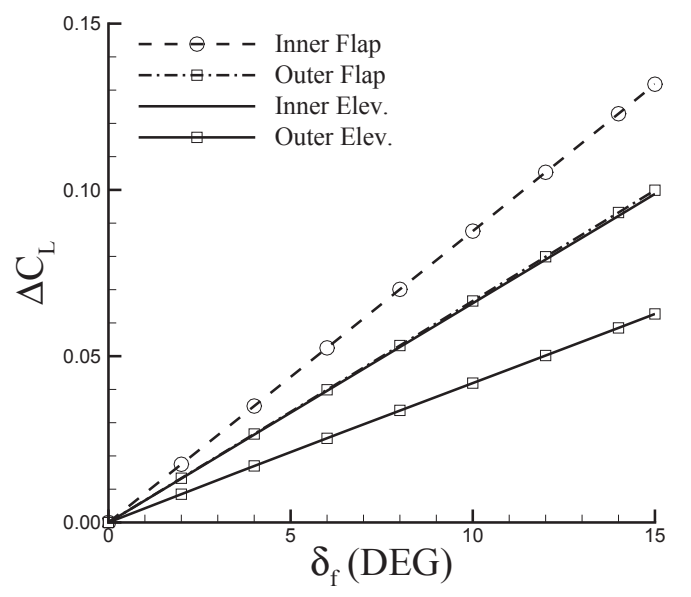

(a)

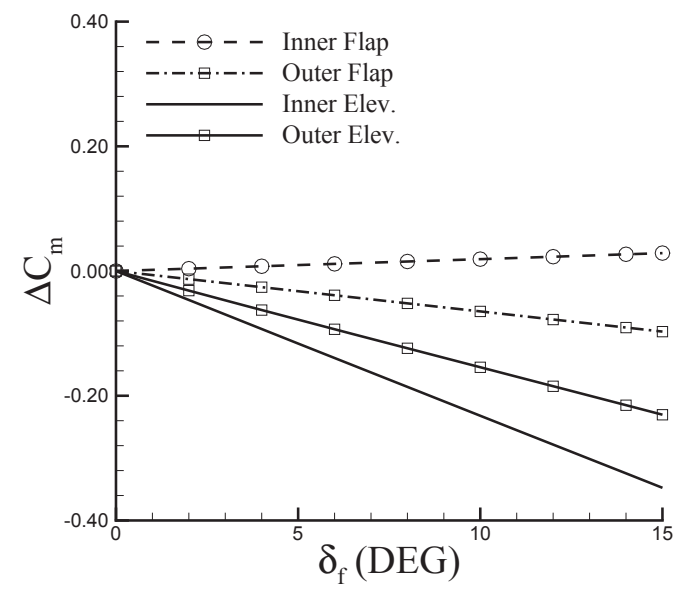

(b)

Figure 11. The increments of lift and pitching moment coefficients for the deflection of elevator, inner and outer flaps at Mach number 0.1 and zero angles of incidence and side-slip

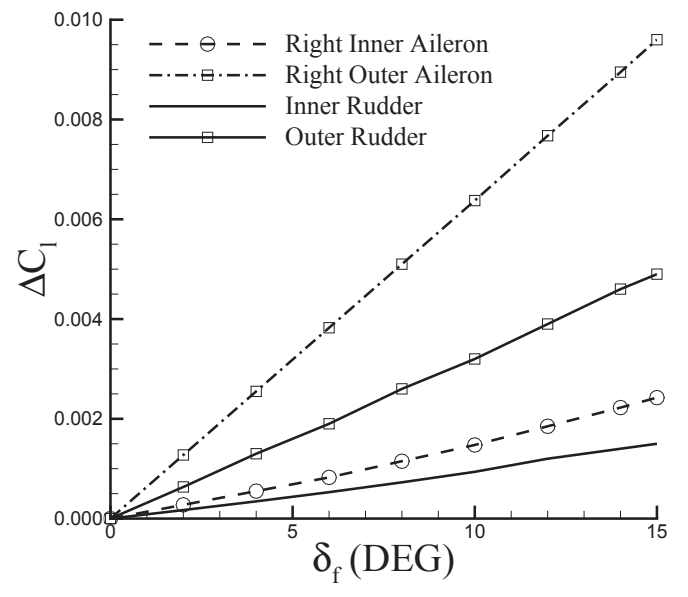

(a)

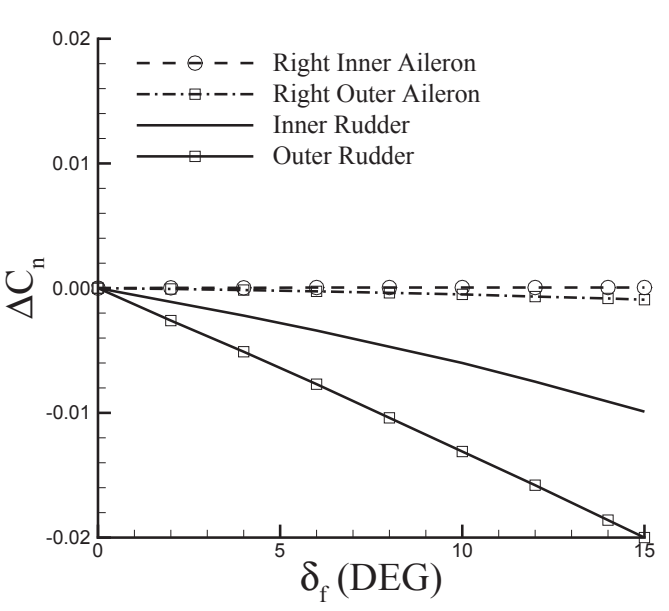

(b)

Figure 12. The control effectiveness for the deflection of inner and outer aileron and rudders at Mach number 0.1 and zero angles of incidence and side-slip

14. Figure shows that for considered Mach number of 0.75 , Tornado predictions for control aerodynamics underestimate the magnitude of changes calculated from EDGE.

\section{A. Ranger 2000}

The table (angle of attack, Mach number and side-slip angle) was generated from scratch using sampling. A 500 entry table was first generated using the PMB solver for 65 calculations. The generated table describes the underlying behaviour of aerodynamic forces and moments. Next, the tables for varying angle of attack, Mach number and control surface deflections were generated. Twelve additional samples for each configuration at non-zero deflection angles were calculated. The values at these samples were then used to increment the static table values using Co-Kriging.

Wind tunnel data for the aerodynamic coefficients are available at low speed. The trend of control power with angle of attack from CFD and experimental data is compared in Fig. 15(c)-(e). The flight speed 


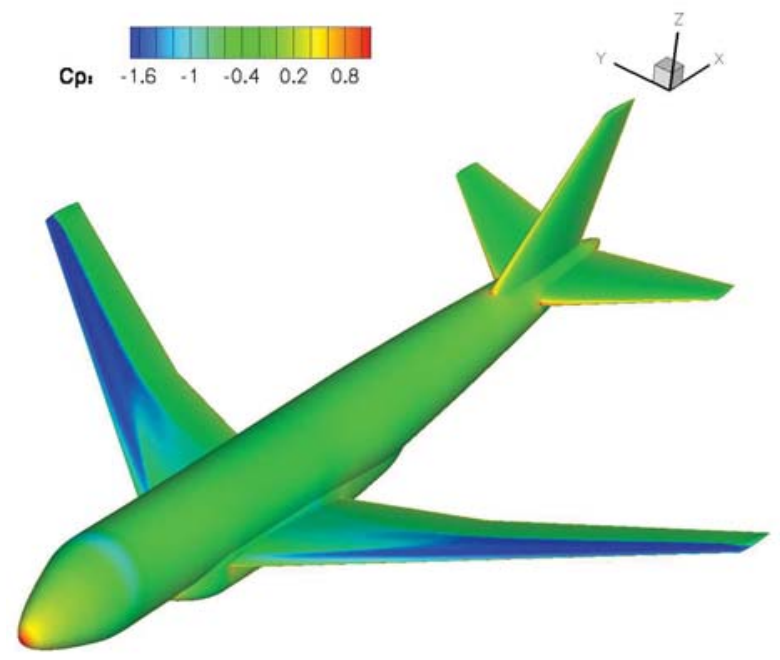

(a) Test Case 1

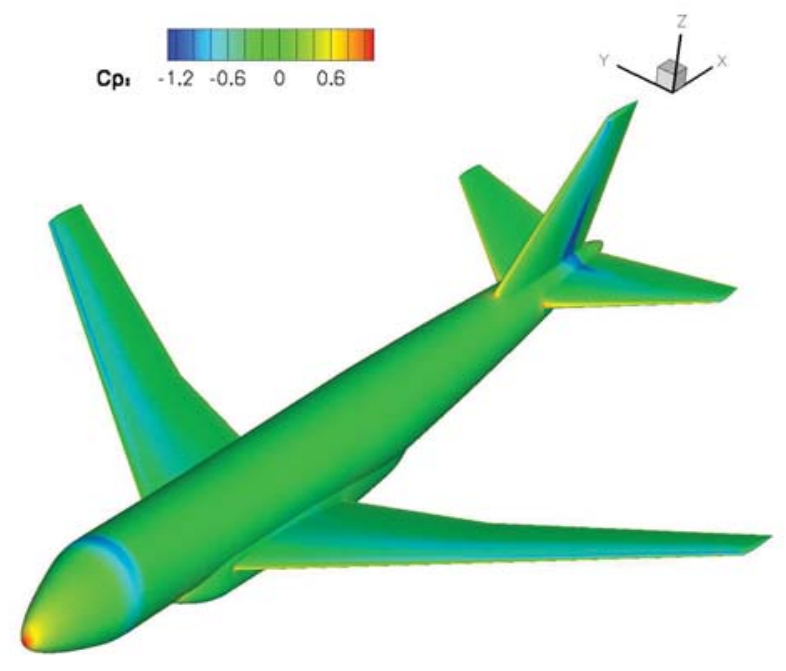

(c) Test Case 3

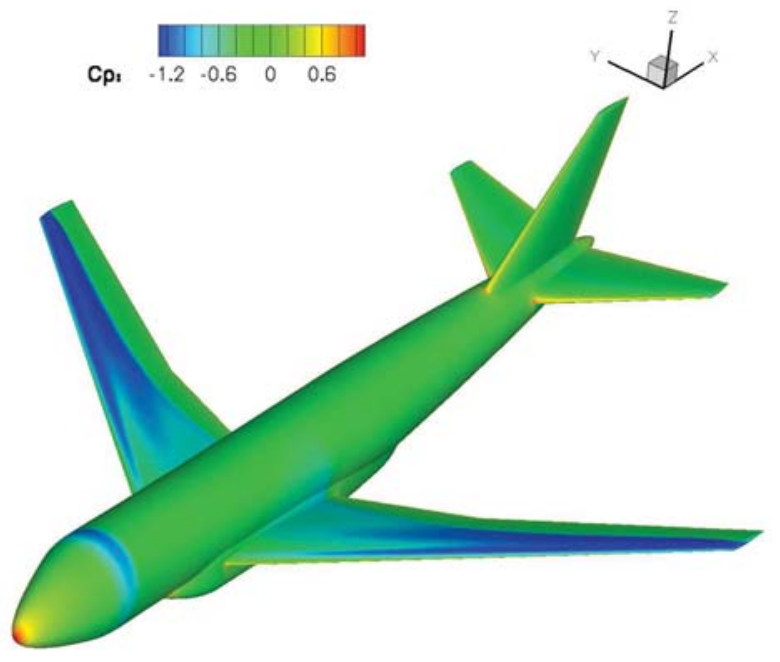

(b) Test Case 2

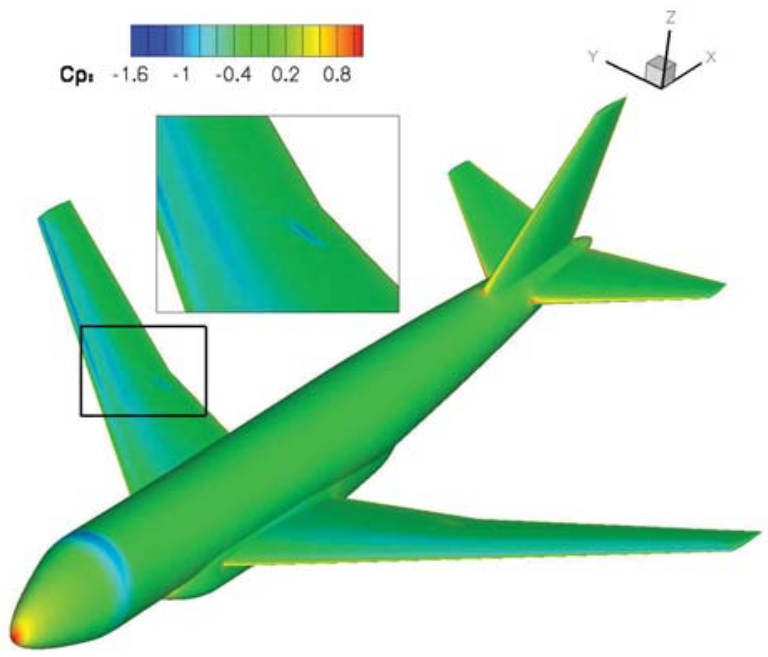

(d) Test Case 4

Figure 13. Surface pressure distribution for the B747 model geometry for Test Case $1\left(\alpha=6.0^{\circ}\right.$ and $\left.M=0.8\right)$ and Test Case $2\left(\alpha=3.6^{\circ}\right.$ and $\left.M=0.85\right)$; Test Case $3\left(\alpha=1.0^{\circ}, M=0.8\right.$ and $\left.\delta_{\text {rud }}=10.0^{\circ}\right)$ and Test Case 4 $\left(\alpha=1.0^{\circ}, M=0.8\right.$ and $\left.\delta_{a i l}=10.0^{\circ}\right)$ feature deflection of rudder and inner ailerons, respectively

is $\mathrm{M}=0.25$ with zero side-slip angle for all the figures. The results of two elevator surface deflections are presented, while the angle of deflection for rudder and aileron is +9 degree. The results are in good agreement with measured data. Difference are possibly due to using the blended approach for the treatment of surface deflections. Finally, the mode shape approach for modeling elevator, aileron and rudder is presented in Fig. 16.

\section{Control Allocation Examples}

The generated aero tables are implemented in the control allocation problem. These tables have each the aerodynamic moment coefficients for different control surface deflections between upper and lower limits. For each iteration in the solution algorithm, the moment coefficients are interpolated from each table, added together and compared with desired moments. The algorithm iterates until a convergence criteria is met. For illustration purposes, two examples are considered: 


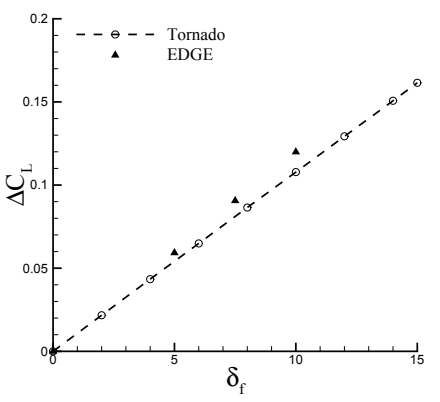

Elevator control effectiveness

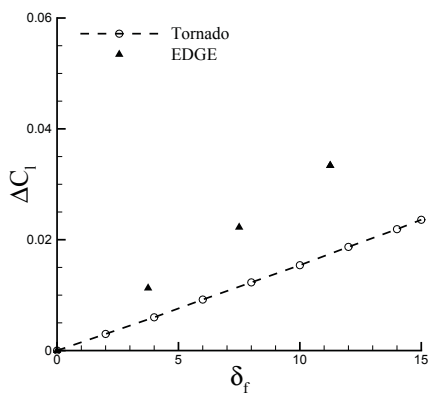

Aileron control effectiveness

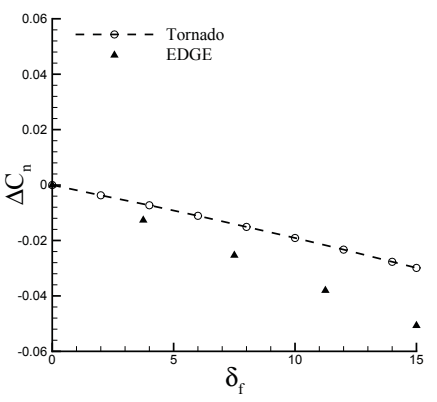

Rudder control effectiveness

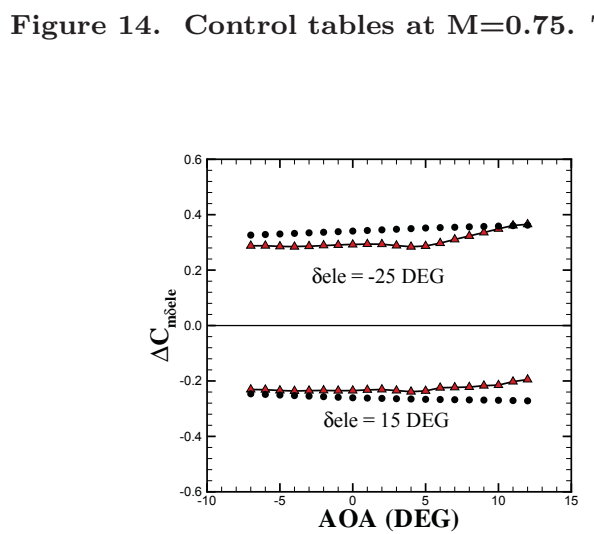

Elevator control effectiveness

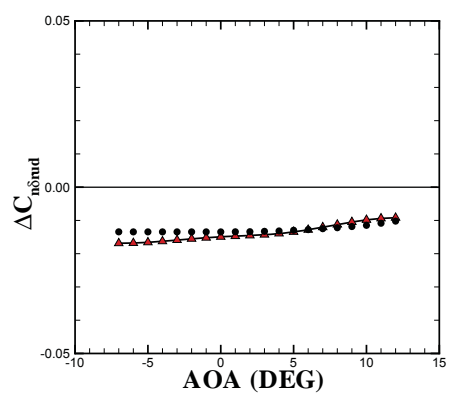

Rudder control effectiveness

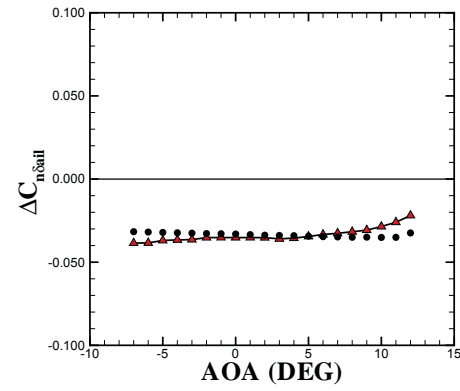

Aileron control effectiveness denote experiments. 


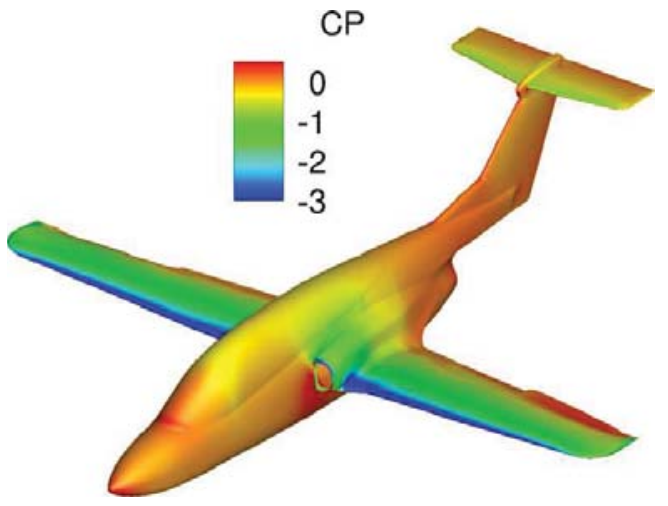

(a)

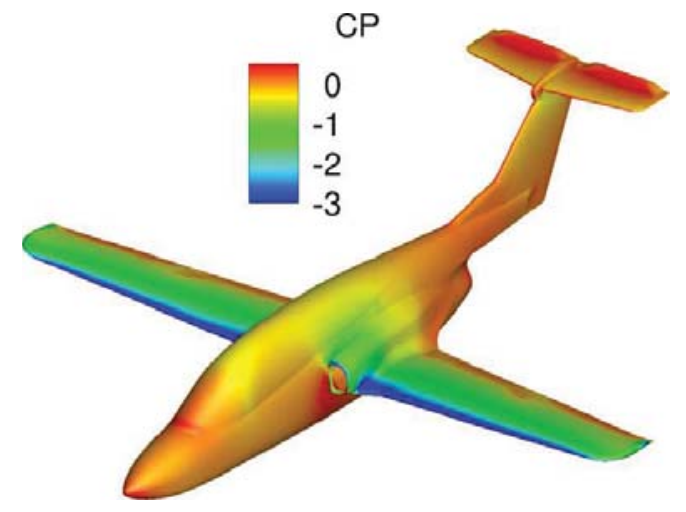

(c)

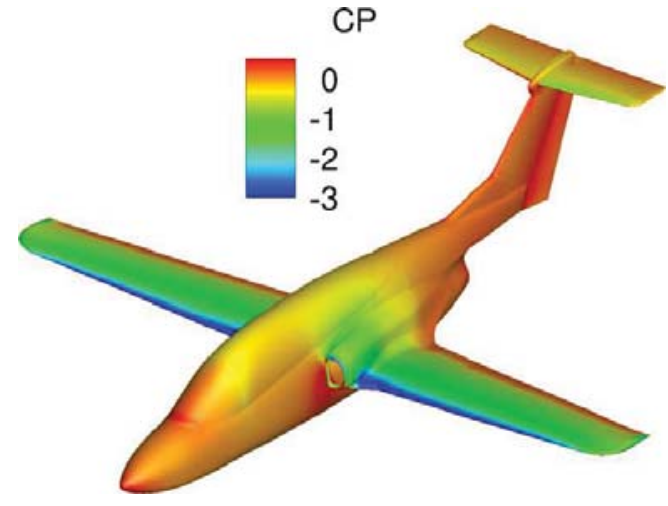

(b)

Figure 16. Surface pressure distribution for the Ranger- The angle of attack and Mach number are $12^{0}$ and 0.3 for all cases. Figure (a) shows $15^{0}$ aileron deflection. In Fig. (b) rudder is moved $17.5^{0}$. Fig. (c) shows the $\mathbf{- 2 5} 5^{0}$ elevator deflection angle

\section{A. The Zero-moment Example}

The problem is to find an optimal set of controls for B747 aircraft in order to have zero moment in each direction. The Tornado look-up tables are used with constrained controllers: left/right inner flap, left/right inner aileron, left/right outer flap, left/right outer aileron, left/right inner elevator, left/right outer elevator and inner and outer rudders. All these surfaces generate moments in all three aircraft body axis. The solution of having zero moment in each direction is:

$$
\left[\begin{array}{c}
0.5710 \\
0.5590 \\
0.5492 \\
0.4433 \\
0.5737 \\
0.6663 \\
0.4252 \\
0.3517 \\
0.2284 \\
-0.7252 \\
-0.2080 \\
0.2078 \\
0.3451 \\
-0.1290
\end{array}\right] \quad \text { corresponding to }\left\{\begin{array}{l}
\text { right inner flap } \\
\text { left inner flap } \\
\text { right inner aileron } \\
\text { left inner aileron } \\
\text { right outer flap } \\
\text { left outer flap } \\
\text { right outer aileron } \\
\text { left outer aileron } \\
\text { right inner elevator } \\
\text { left inner elevator } \\
\text { right outer elevator } \\
\text { left outer elevator } \\
\text { inner rudder } \\
\text { outer rudder }
\end{array}\right\}
$$


All flaps in the wing have positive deflections, i.e., trailing edge down. The inner and outer elevator segments operate asymmetrically. The inner rudder is positive, i.e., trailing edge left, while the outer rudder trailing edge is moved right.

\section{B. Rolling Only Example}

In this example, the Ranger 2000 aircraft with generated Euler tables is considered. The constrained controllers are: right/left elevator, right/left aileron and rudder. Under the constraints given in Table 3, we are looking for the controls that maps to desired moment of $C_{l}=4.0 \times 10^{-2}$ and $C_{n}=0$. Fig. 17 shows the optimum values of control deflections per iteration. The values remain nearly constant after 2,500 iteration. The solution is found in order of seconds.

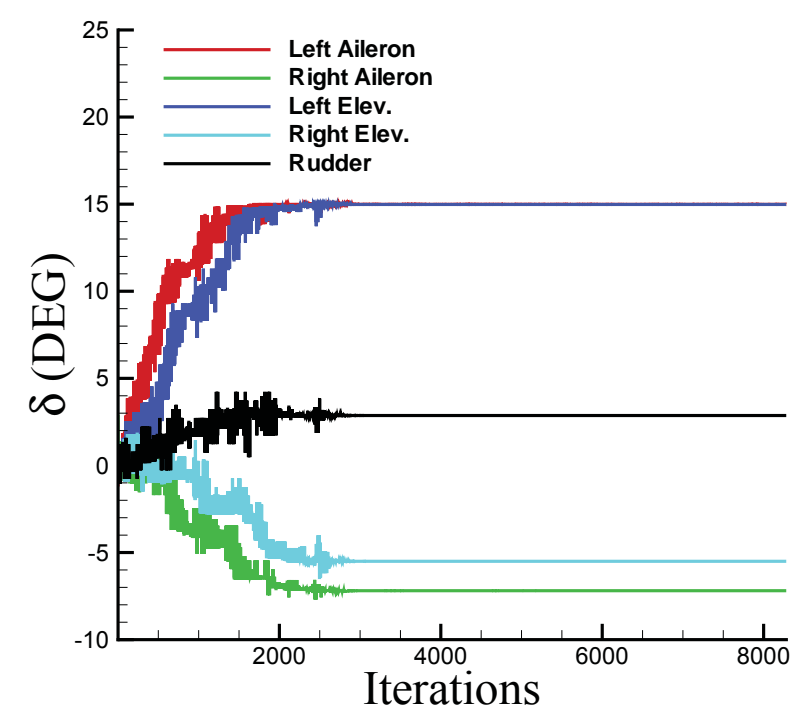

Figure 17. Rolling only Example

The left aileron and elevator are deflected to maximum limit. The right aileron and elevator are negative trailing edge down, while the rudder is positive trailing edge left.

\section{Conclusions}

The paper describes a framework of generating tabular aero data for control allocation problem by using CFD calculations. The CFD solvers used are a potential solver, an unstructured-grid and a structured-grid Euler solver. The treatment of control surface deflection in each solver was described for automatic generation of aero tables. The potential solver rotates the trailing edge of flaps around the trailing edge for any number of flaps over a wide range of deflection angles. However, the aerodynamic control effectiveness is linear with respect to deflection angles and calculations are limited for high speed and angle of attack flight conditions. The EDGE code with transpiration boundary conditions was used for high speed aerodynamics. In this approach, instead of moving the grid, the wall velocity component normal to the actual deflected surface is prescribed, however, this limits the amount of maximum and minimum deflection angles. The PMB solver using mode shapes was also used to generate aerodata. The transfinite interpolation was used to distribute the surface grid point deflections specified from mode shapes to the volume grid. This approach underestimates the aerodynamics of large deflection of control surfaces since the inboard and outboard sections of moving surface are fixed and not rotated.

Sampling and Data fusion was used to allow the generation of the aerodynamic tables in a feasible number of CFD calculations. The aerodata were generated for the reduced geometry description of Boeing 747-100 and Ranger 2000, while the CAD and mesh models of the former case were created automatically 
using SUMO. For each control surface, fifteen samples were only generated using Latin hypercubic sampling. The values at these samples were then used to increment the baseline table values using Co-Kriging. The validation was performed against any available experimental data. The application of CFD to modeling aerodynamics of surface deflections provide quite accurate results.

The results in this paper demonstrate that the automated calculation of large tables of control aerodynamics is now possible using physics based aerodynamic simulation data. This allows design of control laws of unstable and unconventional aircraft configurations early in the design stage. The future work will explore the used CFD methods and other methods such as overset-grid for the design of an aircraft autopilot system with a control allocator.

\section{Acknowledgments}

Computer time for Ranger calculations was provided through the UK Applied Aerodynamics Consortium (UKAAC) under Engineering and Physical Sciences Research Council grant EP/F005954/1. Also, the authors would like to thank Dr. Stefan Hitzel at EADS to provide the Ranger geometry and experimental data. This work was supported by the SimSAC project under funding from the Sixth Framework programme of the European Union. The financial support is gratefully acknowledged.

\section{References}

\footnotetext{
${ }^{1}$ Oh, J.-H., Jamoom, M. B., McConley, M. W., and Feron, E., "Solving Control Allocation Problems Using Semidefinite Programming," Journal of Guidance, Control, and Dynamics, Vol. 22, No. 3, 1999, pp. 494-497.

${ }^{2}$ Buffington, J. M., Enns, D. F., and Teel, A. R., "Control Allocation and Zero Dynamics," Journal of Guidance, Control, and Dynamics, Vol. 21, No. 3, 1998, pp. 458-464.

${ }^{3}$ Bolender, M. A. and Doman, D. B., "Method for Determination of Nonlinear Attainable Moment Sets," Journal of Guidance, Control, and Dynamics, Vol. 27, No. 5, 2004, pp. 907-914.

${ }^{4}$ Durham, W. C., "Constrained Control Allocation," Journal of Guidance, Control, and Dynamics, Vol. 16, No. 4, 1993, pp. $717-725$

${ }^{5}$ Durham, W. C., "Constrained Control Allocation: Three-Moment Problem," Journal of Guidance, Control, and Dynamics, Vol. 17, No. 2, 1994, pp. 330-336.

${ }^{6}$ Bodson, M., "Evaluation of Optimization Methods for Control Allocation," Journal of Guidance, Control, and Dynamics, Vol. 25, No. 4, 2002, pp. 703-711.

${ }^{7}$ Johansen, T. A., Fossen, T. I., and Tndel, F., "Efficient Optimal Constrained Control Allocation via Multiparametric Programming," Journal of Guidance, Control, and Dynamics, Vol. 28, No. 3, 2005, pp. 506-515.

${ }^{8}$ Page, A. and Steinberg, M., "A Closed-Loop Comparison of Control Allocation Methods," AIAA Guidance, Navigation, and Control Conference and Exhibit, AIAA 2000-4538, 2000.

${ }^{9}$ Enns, D. F., "Control Allocation Approaches," AIAA Guidance, Navigation, and Control Conference and Exhibit, 1998.

${ }^{10}$ Bolender, M. A. and Doman, D. B., "Non-Linear Control Allocation Using Piecewise Linear Functions: A Linear Programming approach," Air Force Research Laboratory report, AFRL-VA-WP-TP-2004-303, February, 2004.

${ }^{11}$ Rogers, S. E., Aftomis, M. J., Pandya, S. A., Chaderjian, N. M., Tejnil, E. T., and Ahmad, J. U., "Automated CFD Parameter Studies on Distributed Parallel Computers," 16th AIAA Computational Fluid Dynamics Conference, Orlando, Florida, AIAA 2003-4229, 2003.

${ }^{12}$ Ghoreyshi, M., Badcock, K. J., and Woodgate, M. A., "Accelerating the Numerical Generation of Aerodynamic Models for Flight Simulation," Journal of Aircraft, Vol. 46, No. 3, 2009, pp. 972-980.

${ }^{13}$ Von Kaenel, R., Rizzi, A., Oppelstrup, J., Goetzendorf-Grabowski, T., Ghoreyshi, M., Cavagna, L., and Berard, A., "CEASIOM: Simulating Stability \& Control with CFD/CSM in Aircraft Conceptual Design," Proceedings of the 26th Congress of the International Council of the Aeronautical Sciences, 2008.

${ }^{14}$ Da Ronch, A., "CFD Start Documentation," SimSAC Official Deliverable, D 3.2-6, 2009.

${ }^{15}$ Khrabrov, A., Kolino, K., Glazkov, S., Sidoryuk, M., Bolyrev, S., and Anoshin, J., "Review of Steady and Unsteady Panel Codes and Tier I Simulation of Aircraft Stability and Control Characteristics Using Vortex Lattice Method," SimSAC Official Deliverable, D 3.2-1, 2007.

${ }^{16}$ Da Ronch, A., McFarlane, C., Beaverstock, C., Oppelstrup, J., Zhang, M., and Rizzi, A., "Benchmarking CEASIOM Software to Predict Flight Control and Flying Qualities of the B-747," Proceedings of the 27th Congress of the International Council of the Aeronautical Sciences, ICAS 2010-5.10.1, Nice, France, 19-24 September, 2010.

${ }^{17}$ Anderson, J. D., "Modern Compressible Flow," McGraw Hill, 1990.

${ }^{18}$ Melin, T., "TORNADO: a Vortex-Lattice MATLAB Implementation for Linear Aerodynamic Wing Applications," Master Thesis, Royal Insitute of Technology (KTH), Department of Aeronautics, Sweden, December, 2000.

${ }^{19}$ Eliasson, P., "EDGE, a Navier-Stokes Solver for Unstructured Grids," FOI Report, FOI-R-0298-SE, 2005.

${ }^{20}$ Badcock, K. J., Richards, B. E., and Woodgate, M. A., "Elements of Computational Fluid Dynamics on Block Structured Grids using Implicit Solvers," Progress in Aerospace Sciences, Vol. 36, No. 5, 2000, pp. 351-392.

${ }^{21}$ Boelens, O. J., Badcock, K. J., Elmilgui, A., Abdol-Hamid, K. S., and Massey, S. J., "Comparison of Measured and Block structured Simulation Results for the F-16XL Aircraft," Journal of Aircraft, Vol. 46, No. 2, 2009, pp. 377-384.
} 
${ }^{22}$ Rampurawala, A. M. and Badcock, K. J., "Evaluation of a Simplified Grid Treatment for Oscillating Trailing-Edge Control Surfaces," Vol. 44, 1801 Alexander Bell Drive, Suite 500, Reston, VA 20191-4344, United States, 2007, pp. $1177-1188$. 